\title{
Molecular Mechanisms of Heart Valve Development and Disease
}

\author{
M. Victoria Gomez Stallons, Elaine E. Wirrig-Schwendeman, Ming \\ Fang, Jonathan D. Cheek, Christina M. Alfieri, Robert B. Hinton, \\ and Katherine E. Yutzey
}

\begin{abstract}
The mature heart valves consist of stratified extracellular matrix (ECM) layers, and heart valve disease is characterized by ECM dysregulation and mineralization. There is increasing evidence that regulatory pathways that control heart valve development also are active in disease. In human diseased valves and mouse models, the expression of valve progenitor markers, including Twist1, Msx1/2 and Snail1/2, is induced. Additional markers of osteogenesis, including Runx2, osteocalcin and bone sialoprotein, also are expressed in calcific aortic valve disease (CAVD) in humans and mice. New mouse models have been developed for studies of valve disease mechanisms. Klotho-null mice are a model for premature aging and exhibit calcified nodules in aortic valves with osteogenic gene induction. Osteogenesis Imperfecta mice, bearing a collagen 1a2 mutation, develop features of myxomatous valve disease, including thickening, increased proteoglycan deposition and chondrogenic gene induction. Together, these findings demonstrate specific molecular indicators of valve disease progression, including the identification of early disease markers, which represent potential targets for therapeutic intervention.
\end{abstract}

\section{Keywords}

Heart valve $\bullet$ Embryo $•$ Mouse model $•$ Aortic valve disease

M.V. Gomez Stallons • E.E. Wirrig-Schwendeman • M. Fang • J.D. Cheek • C.M. Alfieri • R.B. Hinton • K.E. Yutzey $(\bowtie)$

Division of Molecular Cardiovascular Biology, The Heart Institute, Cincinnati Children's Hospital Medical Center, 240 Albert Sabin Way, ML 7020, Cincinnati, OH 45229, USA

e-mail: Katherine.Yutzey@cchmc.org

T. Nakanishi et al. (eds.), Etiology and Morphogenesis of Congenital Heart Disease,

DOI 10.1007/978-4-431-54628-3_18 


\subsection{Introduction}

The semilunar and atrioventricular (AV) valves of the heart are made up of highly organized extracellular matrix (ECM) layers populated by quiescent valve interstitial cells (VICs) [1]. In healthy valves, the ECM is compartmentalized into layers composed of collagens, proteoglycans, and elastin, which are maintained by the VICs for proper valve function throughout life (Reviewed in [2]). In diseased valves, the leaflets are thickened as a result of ECM dysregulation and VIC activation. Calcific aortic valve disease (CAVD) includes calcification of the cusps [3], whereas mitral valve prolapse (MVP) is accompanied by increased proteoglycans and myxomatous changes in the leaflets [4]. Currently, the standard treatment for severe heart valve disease is surgical replacement, and new therapies based on molecular mechanisms are needed.

Molecular mechanisms associated with heart valve disease include activation of signaling pathways involved in progenitor specification, cell proliferation, and differentiation of heart valve and bone precursors [5, 6]. We have reported that pediatric and adult diseased valves are characterized by expression of markers of valve mesenchymal and chondrogenic progenitor cells, while adult diseased aortic valves express markers of osteogenic calcification [7]. We also have identified novel mouse models of calcific and myxomatous valve disease [8] that will be useful in determination of the underlying mechanisms driving disease and in development of pharmacologic-based therapies.

\subsection{Heart Valve Development}

Heart valve development in vertebrate embryos begins with the formation of endocardial cushions in the AV canal and outflow tract of the primitive heart tube [5]. The mesenchymal cells of the endocardial cushions originate from the endocardium after an endothelial-to-mesenchymal transition (EMT). Valve progenitors are highly proliferative and migratory and express transcription factors Twist1, Tbx20, Sox9, Msx1/2, and Snai1. The endocardial cushion cells diversify into lineages that express distinct ECM profiles regulated by BMP and FGF signaling [9]. Wnt/ $\beta$-catenin signaling also is active during endocardial cushion maturation, but the specific role for this pathway in valve lineage differentiation is yet to be determined [10]. Valve development continues with the remodeling of the endocardial cushions into thin elongated leaflets composed of stratified ECM, which occurs soon after birth in mice and humans [2]. The ECM layers consist of the collagen-rich fibrosa, proteoglycan-rich spongiosa, and elastin-rich atrialis/ ventricularis [2]. These layers are oriented in the semilunar and AV valves with the elastin layer adjacent to blood flow. While it is likely that hemodynamics has a role in leaflet stratification, the regulatory pathways that control ECM organization and compartmentalization during valve maturation are largely unknown. 


\subsection{Heart Valve Disease}

Heart valve disease can result from congenital malformation or gene mutations, or it may be acquired later in life [1]. The prevalence of heart valve disease increases significantly with age, such that $\sim 10 \%$ of people $>75$ years old have moderate aortic or mitral valve disease [11]. However, the pathogenic mechanisms that drive the development of heart valve disease and that could serve as potential therapeutic targets are not well understood. There is increasing evidence that regulatory pathways that control heart valve and bone development also are active in disease. However, the roles of these pathways in valve pathogenesis and/or repair are not well defined.

\subsubsection{Calcific Aortic Valve Disease (CAVD)}

CAVD is a progressive disease, initially presenting with aortic valve (AoV) thickening (sclerosis) and resulting in valve stenosis and insufficiency later in life [12]. End-stage disease is characterized by the presence of calcific nodules at the hinge region of the AoV, underlying the pathology of CAVD [3]. In an effort to draw parallels between the progression of disease and the underlying molecular mechanisms, pediatric and adult diseased AoV were analyzed for markers of valve development and endochondral bone formation [7]. Activated VICs in both pediatric and adult valves have increased expression of valvulogenic markers Twist1, Msx2, and Sox9. Strikingly, the formation of calcific nodules was found to be an exclusive feature of adult calcified AoV. Furthermore, phosphorylation of Smads1/ $5 / 8$, indicative of active BMP signaling, in addition to expression of osteogenic genes, such as Runx2, was observed only in adult calcified AoV. These findings demonstrate that both pediatric and adult diseased AoV express valvulogenic markers, while adult calcified AoV also express markers of osteogenic calcification. Differential expression of these markers suggests that an osteogenic regulatory mechanism contributes specifically to CAVD.

The incidence of human CAVD strongly correlates with aging, which is an independent risk factor for AoV disease. We have recently identified $\mathrm{Klotho}^{-/-}$mice, a model of premature aging, as a novel mouse model of CAVD [8]. Notably, $\mathrm{Klotho}^{-/-}$mice develop calcific nodules at the hinge region of the fibrosa side of the AoV (Fig. 18.1a, b), similar to human CAVD. In these mice, calcification occurs independent of inflammation and cusp thickening, providing initial evidence for a valve-intrinsic molecular mechanism for age-related calcification common in elderly patients. Klotho ${ }^{-/-} \mathrm{AoV}$ have increased expression of osteogenic factors Runx 2 and Osteopontin, in addition to increased expression of chondrogenic factors Sox9 and Colloal, consistent with an osteochondrogenic-like mechanism of disease (Fig. 18.1e). Increased activation of $\mathrm{pSmad1/5/8}$ also precedes calcification in the Klotho $^{-/-}$mice, and inhibition of BMP signaling represents an attractive new therapeutic approach for CAVD. 

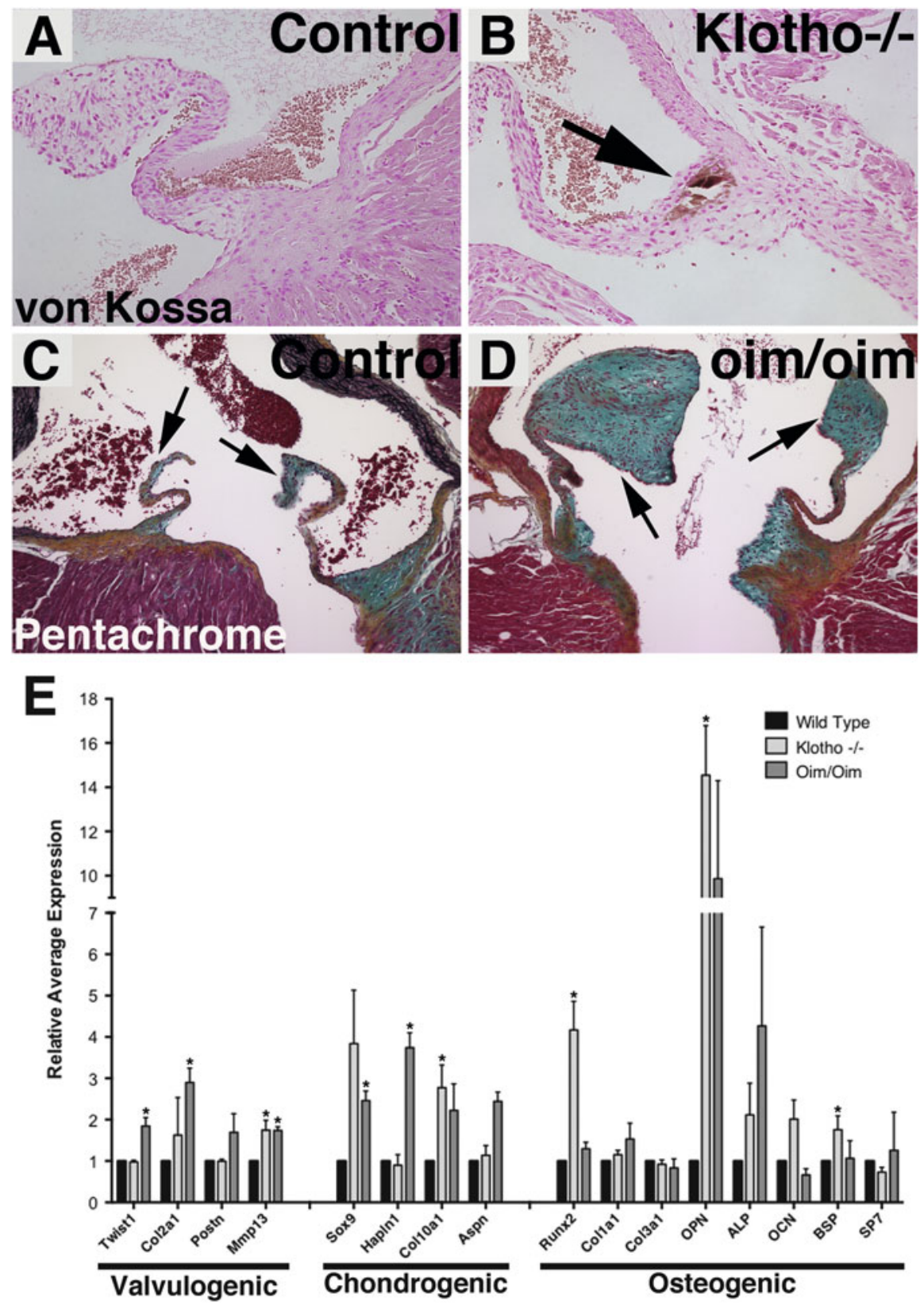

Fig. 18.1 Valvulogenic, chondrogenic, and osteogenic programs are induced in mouse models of calcific $\left(\right.$ Klotho $\left.^{-1}\right)$ and myxomatous $($ Oim/Oim $)$ valve disease. Klotho ${ }^{-/-}$mice $(\mathbf{b})$ exhibit AoV nodular calcification (arrows), as compared to wild-type littermate controls (a) at 6 weeks of age, as detected by von Kossa staining. Oim/Oim AoV cusps (d) exhibit distal thickening and increased proteoglycan deposition (aqua, arrows), compared to WT littermates (c) at 9 months of age as observed by Movat's pentachrome staining. Expression of genes involved in valvulogenesis, chondrogenesis, and osteogenesis was examined by qRT-PCR of RNA isolated from Klotho ${ }^{-/}$ 


\subsubsection{Myxomatous Valve Disease}

The most common cause of MVP is myxomatous valve disease, which is defined by pathological thickening of the valve leaflets, primarily due to accumulation of proteoglycans [4]. This is accompanied by alterations in the distribution of ECM components, such as disrupted collagen fiber organization and elastic fiber fragmentation. The pathogenesis of MVP is not well understood; however, MVP is often linked to connective tissue disorders or specific mutations in ECM genes, supporting the concept that defects originating during valve development could underlie adult disease.

While myxomatous disease most commonly affects the mitral valves, myxoid AoV have been reported. Osteogenesis imperfecta murine (Oim) have a spontaneous mutation in the Colla2 gene and display bone fragility characteristic of human osteogenesis imperfecta (OI) [13]. Interestingly, humans with OI or Colla2 mutations have a predisposition to AoV disease [14]. Likewise, the AoV of Oim/ Oim mice exhibit distal cusp thickening and increased proteoglycan accumulation, characteristic of myxomatous valve disease (Fig. 18.1c, d) [8]. Furthermore, the Oim/Oim mice have increased expression of valve progenitor markers Twistl, Col2al, Mmp13, Sox9, and Hapln1, in addition to increased CollOal and Asporin expression (Fig. 18.1e). These changes in gene expression are consistent with increased proteoglycan accumulation and cartilage gene induction, which are key features of myxomatous disease.

\subsection{Signaling Pathways in Heart Valve Development and Disease}

Similar to heart valve and bone development, studies of human explanted valves implicate BMP, Notch, and Wnt signaling pathways in the progression of CAVD. Thus, heart valve disease shares signaling networks with valve and bone developmental pathways. Together, these studies demonstrate that activation of both BMP and Wnt signaling correlates with progression of CAVD $[15,16]$. On the other hand, loss-of-function mutations in NOTCHI are associated with bicuspid aortic valve (BAV) and CAVD, in humans and mice, suggesting an inhibitory function for the Notch pathway in valve calcification [17]. Human genetic conditions including Marfan syndrome and Loeys-Dietz syndrome lead to myxomatous mitral valve disease and are associated with increased TGF- $\beta$ signaling $[18,19]$. However, the specific mechanisms by which these different pathways contribute to the development and progression of heart valve disease remain unknown.

Fig. 18.1 (continued) and Oim/Oim mice aortic valves relative to wild-type littermate controls (e). Normalized values are shown as average fold changes compared to wild-type group set at 1.0.

* is $p$-value $\leq 0.05$ calculated by paired student's $t$-test 


\subsection{Future Directions and Clinical Implications}

Klotho $^{-/-}$and Oim/Oim mice are novel mouse models of CAVD and myxomatous valve disease that will be useful for determination of the underlying pathogenic mechanisms driving valve disease. Understanding how signaling networks contribute to disease will likely have a significant impact on clinical outcomes, since knowledge gained from these studies will allow for the development and design of new drugs/treatments for patients with valve disease.

Acknowledgments This work was supported by funding from NIH HL082716, HL094319, HL114682 (K.E.Y), F32HL110390 (E.E.W.), and the American Heart Association-Great Rivers Affiliate (M.V.G. and M.F.).

Open Access This chapter is distributed under the terms of the Creative Commons AttributionNoncommercial 2.5 License (http://creativecommons.org/licenses/by-nc/2.5/) which permits any noncommercial use, distribution, and reproduction in any medium, provided the original author(s) and source are credited.

The images or other third party material in this chapter are included in the work's Creative Commons license, unless indicated otherwise in the credit line; if such material is not included in the work's Creative Commons license and the respective action is not permitted by statutory regulation, users will need to obtain permission from the license holder to duplicate, adapt or reproduce the material.

\section{References}

1. Lincoln J, Yutzey KE. Molecular and developmental mechanisms of congenital heart valve disease. Birth Defects Res A Clin Mol Teratol. 2011;91:526-34.

2. Hinton RB, Yutzey KE. Heart valve structure and function in development and disease. Annu Rev Physiol. 2011;73:29-46.

3. Otto CM, Kuusisto J, Reichenbach DD, Gown AM, O'Brien KD. Characterization of the early lesion of 'degenerative' valvular aortic stenosis. Histological and immunohistochemical studies. Circulation. 1994;90:844-53.

4. Guy TS, Hill AC. Mitral valve prolapse. Annu Rev Med. 2012;63:277-92.

5. Combs MD, Yutzey KE. Heart valve development: regulatory networks in development and disease. Circ Res. 2009;105:408-21.

6. Rajamannan NM, Subramaniam M, Rickard DJ, Stock SR, Donovan J, Springett M, Orszulak T, Fullerton DA, Tajik AJ, Bonow RO, et al. Human aortic valve calcification is associated with an osteoblast phenotype. Circulation. 2003;107:2181-4.

7. Wirrig EE, Hinton RB, Yutzey KE. Differential expression of cartilage and bone-related proteins in pediatric and adult diseased aortic valves. J Mol Cell Cardiol. 2011;50:561-9.

8. Cheek JD, Wirrig EE, Alfieri CM, James JF, Yutzey KE. Differential activation of valvulogenic, chondrogenic, and osteogenic pathways in mouse models of myxomatous and calcific aortic valve disease. J Mol Cell Cardiol. 2012;52:689-700.

9. Lincoln J, Alfieri CM, Yutzey KE. BMP and FGF regulatory pathways control cell lineage diversification of heart valve precursor cells. Dev Biol. 2006;292:290-302.

10. Alfieri CM, Cheek J, Chakraborty S, Yutzey KE. Wnt signaling in heart valve development and osteogenic gene induction. Dev Biol. 2010;338:127-35.

11. Nkomo VT, Gardin JM, Skelton TN, Gottdiener JS, Scott CG, Enriquez-Sarano M. Burden of valvular heart diseases: a population-based study. Lancet. 2006;368:1005-11. 
12. Otto CM. Valvular aortic stenosis: disease severity and timing of intervention. J Am Coll Cardiol. 2006;47:2141-51.

13. Chipman SD, Sweet HO, McBride DJ, Davisson MT, Marks SC, Shuldiner AR, Wenstrup RJ, Rowe DW, Shapiro JR. Defective proa2(I) collagen synthesis in a recessive mutation in mice: a model of human osteogenesis imperfecta. Proc Natl Acad Sci U S A. 1993;90:1701-5.

14. Bonita RE, Cohen IS, Berko BA. Valvular heart disease in osteogenesis imperfecta. Echocardiography. 2010;27:69-73.

15. Ankeny RF, Thourani VH, Weiss D, Vega JD, Taylor WR, Nerem RM, Jo H. Preferential activation of SMAD1/5/8 on the fibrosa endothelium in calcified human aortic valves association with low BMP antagonists and SMAD6. PLoS ONE. 2011;6:e20969.

16. Caira FC, Stock SR, Gleason TG, McGee EC, Huang J, Bonow RO, Spelsberg TC, McCarthy PM, Rahimtoola SH, Rajamannan NM. Human degenerative valve disease is associated with up-regulation of low-density lipoprotein-related protein 5 receptor-mediated bone formation. J Am Coll Cardiol. 2006;47:1707-12.

17. Garg V, Muth AN, Ransom JF, Schluterman MK, Barnes R, King IN, Grossfeld PD, Srivastava D. Mutations in NOTCH1 cause aortic valve disease. Nature. 2005;437:270-4.

18. Dietz HC, Cutting GR, Pyeritz RE, Maslen CL, Sakai LY, Corson GM, Puffenberger EG, Hamosh A, Nanthakumar EJ, Curristin SM, et al. Marfan syndrome caused by a recurrent de novo missense mutation in the fibrillin gene. Nature. 1991;352:337-9.

19. Loeys BL, Chen J, Neptune ER, Judge DP, Podowski M, Holm T, Meyers J, Leitch CC, Katsanis N, Sharifi N, et al. A syndrome of altered cardiovascular, craniofacial, neurocognitive and skeletal development caused by mutations in TGFBR1 or TGFBR2. Nat Genet. 2005;37:275-81. 